\begin{tabular}{l|l} 
REVISTA & $\begin{array}{l}\text { Revista Educación } \\
\text { ISSN: } 0379-7082 \\
\text { ISSN: 2215-2644 } \\
\text { revedu@gmail.com } \\
\text { Universidad de Costa Rica } \\
\text { Costa Rica }\end{array}$
\end{tabular}

\title{
Reflexiones pedagógicas sobre la enseñanza del Derecho
}

\author{
Gatgens Gómez, Erick \\ Reflexiones pedagógicas sobre la enseñanza del Derecho \\ Revista Educación, vol. 44, núm. 2, 2020 \\ Universidad de Costa Rica, Costa Rica \\ Disponible en: http://www.redalyc.org/articulo.oa?id=44062184008 \\ DOI: https://doi.org/10.15517/revedu.v44i2.36988
}

Esta obra está bajo una Licencia Creative Commons Atribución-NoComercial-SinDerivar 3.0 Internacional. 


\title{
Reflexiones pedagógicas sobre la enseñanza del Derecho
}

\author{
Pedagogical Reflections about Teaching Law \\ Erick Gatgens Gómez \\ Universidad de Costa Rica, Costa Rica \\ erick.gatgens@penalistascr.com \\ (iD http://orcid.org/0000-0003-1994-3896
}

DOI: https://doi.org/10.15517/revedu.v44i2.36988

Redalyc: http://www.redalyc.org/articulo.oa?id=44062184008

Recepción: 11 Junio 2019

Aprobación: 20 Febrero 2020

\section{Resumen:}

El presente ensayo plantea un cambio de perspectiva en el modelo educativo, a fin de que este se efectúe de manera bidireccionaldialéctica, entre el profesorado y el alumnado, en donde el primero innova en sus clases y reta intelectualmente al alumnado por medio de la resolución de casos, estimulándolo a ejecutar preguntas, fortaleciendo la oralidad, y evaluando el aprendizaje por medio de la argumentación-fundamentación válida, lógica y racional, en la cual el y la docente ayuda al estudiante a percatarse de sus errores, en vez de darle la respuesta correcta ante el primer yerro del estudiantado. Se propone una participación esencialmente activa del cada estudiante.

Palabras clave: Pedagogía, Perspectiva, Actividades, Participación activa del estudiantado, Enseñanza, Aprendizaje significativo, Educación, Conocimiento, Construcción, Razonamiento, Derecho.

\section{Abstract:}

This study proposes a change in perspective with regards to the educational model so that teacher-student interaction may be conducted in a bidirectional-dialectical manner. Teachers must be an innovative and intellectually challenge students through case resolution, encouraging them to ask questions, strengthening their oral skills and assess learning through argumentation as well as logical, rational and valid reasoning. Teachers should help students identify their errors instead of providing the correct answer immediately as soon as a student makes a mistake. Teachers should also expect student participation to be active.

KeYwords: Pedagogy, Perspective, Activities, Active Student Participation, Student, Teaching, Significative Learning, Education, Knowledge, Construction, Reasoning, Law.

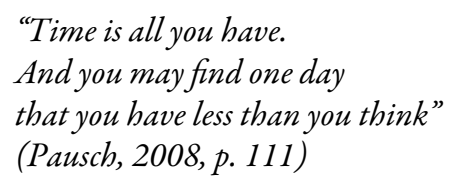

\section{EL OBJETO DE ESTAS REFLEXIONES}

Estas reflexiones se escriben a propósito del Congreso Académico de la Facultad de Derecho, el cual tuvo lugar del 16 al 23 de abril de 2016. El primer día indicado me correspondió impartir la charla objeto de estas reflexiones, en el Auditorio de la Sede de Occidente de la Universidad de Costa Rica. Por lo tanto, el presente artículo recoge -en parte- lo expuesto ese día.

La primera pregunta que debemos responder es sobre qué tópicos giran estas reflexiones. Ante lo cual contestaríamos, en general, que se trata de un análisis sobre pedagogía en la enseñanza del Derecho. Y, ¿qué es la pedagogía? Según De Alcántara (1999), la pedagogía es el conjunto de los principios que presiden a la educación, así como de las reglas o medios de aplicarlos en la práctica, es decir, es al mismo tiempo la ciencia 
y el arte de la educación. En otras palabras, Ruiz (1904) estima que la pedagogía es "el arte científico de enseñar" (p. 8).

Ahora bien, ¿qué es la educación?; y ¿qué es la enseñanza? La educación es "el intento de hacer aflorar (hacia afuera) lo que llevamos dentro, un descubrir de capacidades” (Farfán, Kcomt, Leiva, More, Quevedo, Rodríguez citados por Picardo, Escobar y Balmore, 2005, p. 92.)

La enseñanza, según el diccionario de la Real Academia (1994), tiene las siguientes acepciones:

...2. f. Sistema y método de dar instrucción; 3. f. Ejemplo, acción o suceso que sirve de experiencia, enseñando o advirtiendo cómo se debe obrar en casos análogos; 4. f. pl. Conjunto de conocimientos, principios, ideas, etc., que se enseñan a alguien... (p. 845). [El resaltado es del autor]

Esta última acepción pone de nuevo énfasis, en la labor del profesorado.

Sin embargo, resulta necesario preguntarnos si ¿en la enseñanza y, en general, en la educación es relevante únicamente la labor del y la docente?

Y si no fuera así, ¿qué le corresponde hacer al docente y qué le corresponde hacer al estudiante?

Estamos acostumbrados a ver el proceso de educación-enseñanza en forma lineal, unilateral y no en forma bidireccional y bilateral; pues hemos asumido por mucho tiempo que esos procesos van únicamente en una sola dirección; es decir, la persona docente tienen un rol activo de ilustrar al alumnado, de enseñarle al alumnado y por su parte, el alumnado debe asumir únicamente un rol pasivo, de poner atención, de escuchar y de tomar apuntes, para luego, en sus hogares, aprenderse de memoria todos los contenidos impartidos por el profesorado.

Estimo, sin embargo, que lo fundamental es centrarse en el aprendizaje. El aprendizaje es, según el diccionario de la Real Academia (1994) “...la acción y efecto de aprender algún arte u oficio, así como la adquisición por la práctica de una conducta duradera” (p. 173). Aprender no guarda una relación muy estricta con enseñar. Existen diferentes modos o formas de aprendizaje. Capella (2004) distingue entre un aprendizaje por shock, un aprendizaje de mantenimiento y un aprendizaje innovador. Tal y como señala acertadamente este autor: "Que consigas un buen aprendizaje depende fundamentalmente de ti" (pp. 30-34). Lo anterior significa que cada estudiante debe asumir un rol más activo y protagónico en el proceso de enseñanza-aprendizaje, tal y como se describe en las líneas siguientes.

\section{EL NECESARIO CAMBIO DE PERSPECTIVA}

La lección tradicional en la Facultad de Derecho de la Universidad de Costa Rica discurre de manera tal que el profesorado expone los contenidos del programa a las y los estudiantes, o en el peor de los casos, llega a leerlos. Se trata de un contexto unidireccional y lineal. Cada docente recita el contenido y el alumnado debe aprendérselo de memoria, pero ese aprendizaje se olvida y se va sustituyendo por otros contenidos que el estudiantado va recibiendo a lo largo de la carrera, de forma tal que lo común es reproducir, al concluir la carrera, el material almacenado en la memoria reciente es decir, aquellos contenidos memorizados de último.

En esas lecciones tradicionales, se acostumbra a dar uno o más ejemplos, pero a ellos se acude con fines ilustrativos respecto de lo que se está exponiendo.

Como correctamente señala Bain (2004), citando al profesor Chad Richardson que, la retención tiende a ser más segura, cuando somos llevados con ejemplos a formar una conclusión que cuando a nosotros simplemente se nos presenta un concepto y uno o dos ejemplos para ilustrarlo.

Planteamos en este artículo la necesidad imperante de cambiar de perspectiva. Se trata de un cambio en cuanto al modelo pedagógico que se ha venido utilizando tradicionalmente en la enseñanza del Derecho.

Arias, Francis y Marín (2012) señalan que los modelos pedagógicos establecen las pautas, en las cuales discurrirá la formación, al proponer nociones, fundamentos y estrategias, que consideran el contexto, las finalidades, los sujetos, los recursos económicos y materiales, el contenido y el tiempo. 
Diferentes estudios -algunos de los cuales se citarán más adelante- sugieren la necesidad de implementar cambios en la forma tradicional de impartir lecciones.

Resulta necesario dejar atrás ese enfoque y entender el proceso de enseñanza-aprendizaje en una forma distinta, dinámica, interactiva, recíproca, crítica, bilateral y bidireccional, donde el alumnado asuma un rol activo, participativo y además crítico durante las lecciones, lo cual hace surgir una serie de preguntas relevantes, una de ellas, sería ¿cómo lograr ese cambio? ¿Qué aspectos deben introducirse o incorporarse en una lección, para lograr ese cambio? Bueno, de eso tratarán las siguientes reflexiones.

El resultado que se pretende obtener con este cambio de perspectiva, es promover en las y los estudiantes el desarrollo de las habilidades argumentativas necesarias, para poder enfrentarse con casos de la vida real, contando para ello con una base dogmática-teorética sólida.

\section{Aspectos básicos necesarios para el cambio sugerido}

Un requisito fundamental -diríamos sine qua non- tal y como lo señalan Bain (2004) y Capella (2004) es que el profesorado conozca la materia que imparte, extremadamente bien. Deben saber simplificar y clarificar temas complejos. Deben poder pensar acerca de su propio pensamiento en la disciplina, analizar su naturaleza y evaluar su cualidad. Esa capacidad, que se puede denominar pensamiento metacognitivo, constituye un requisito básico (Bain, 2004).

Otro aspecto fundamental consiste, según Bain (2004) en tratar las clases, charlas, conferencias, secciones de discusión, las sesiones basadas en problemas y otros elementos de la enseñanza, como esfuerzos intelectuales tan demandantes e importantes como sus propias investigaciones y estudios.

Un riesgo frecuente al cual nos enfrentamos quienes nos dedicamos a esta labor, es caer en la rutina, en la costumbre de repetir, con uno que otro cambio -muchas veces superficial- los mismos contenidos impartidos en el curso lectivo anterior. Por lo tanto, resulta fundamental innovar, en el sentido de renovar y corregir lo necesario; así cada vez que tengamos que impartir nuevamente un curso, nos preguntemos críticamente, ¿qué podemos mejorar respecto del ciclo lectivo anterior? ¿Qué aspectos del curso debemos modificar para lograr un mejor proceso de enseñanza-aprendizaje?

En esto, debemos tener claro que, como lo reseña Bain (2004) los mejores profesores esperan lo mejor de sus estudiantes y, además confían en ellos. A menudo conversan acerca de sus propios viajes intelectuales, sus ambiciones, triunfos, frustraciones y errores. Asimismo, de cómo descubrieron sus intereses y los mayores obstáculos que debieron enfrentar antes de dominar la materia.

No obstante lo anterior, la pregunta que surge en este contexto es ¿cómo estimular al estudiante para que sea capaz de alcanzar conocimientos perdurables por medio de un proceso de aprendizaje?

\section{EL AMBIENTE NATURAL CRÍTICO DE APRENDIZAJE}

Fundamental para implementar el cambio indicado, resulta poder crear en la clase, lo que Bain (2004) denomina un ambiente natural crítico de aprendizaje. En ese ambiente, las personas aprenden, al confrontar problemas importantes o intrigantes, tareas auténticas que los van a retar a aprehender ideas, repensar sus asunciones y examinar sus modelos mentales de la realidad. ¿En qué consiste ese ambiente? ¿Por qué se hace referencia a un ambiente natural y crítico? Natural porque el estudiantado encuentra las habilidades, hábitos, actitudes e información que está tratando de aprender, integrado en preguntas y tareas que encuentran fascinantes, tareas auténticas que despiertan curiosidad y se vuelven intrínsecamente interesantes; crítico porque el alumnado aprende a pensar críticamente, a razonar de la evidencia, a examinar la cualidad de su razonamiento usando una variedad de criterios intelectuales, a hacer mejoras o progresos mientras piensan, y a hacer preguntas penetrantes y perspicaces acerca del pensamiento de otras personas. 
$\mathrm{Al}$ respecto Schunk (2012) señala que estructurar el ambiente de aprendizaje resulta fundamental para que el estudiantado pueda construir de forma eficaz nuevos conocimientos y habilidades.

Dentro del contexto indicado, Bain (2004) plantea que hacer referencia a ese ambiente incluye la posibilidad de que el alumnado sea autocrítico, es decir, sea crítico de sí mismo. Por su parte, Pausch (2008) señala que siempre tuvo en mente un objetivo muy importante: ayudar a cada estudiante a aprender a juzgarse. Que sea capaz de reconocer sus verdaderas habilidades, a tener un sentido de sus propias fallas; que sean realistas acerca de cómo otros los ven a ellos. Coincido con él, cuando señala que el personal docente le sirven mejor al estudiantado al ayudarle a ser más autorreflexivo. Es decir, desarrollar una habilidad real de evaluarse uno mismo.

A criterio de Bain (2004), algunos profesores crean este ambiente entre las clases magistrales; otros, con discusiones; y aún otros, con estudios de casos, asignación de roles, trabajo de campo o una variedad de otras técnicas. Unos pocos lo crean con un proyecto central que el estudiantado asume, a menudo, trabajando en colaboración con otros miembros de la clase.

Por caso entendemos, siguiendo a Carrió (1995), un problema práctico. En esto es importante recordar que la labor de los abogados es una labor técnica, consiste en usar ciertos medios para alcanzar un resultado a partir de una situación inicial dada. Menester también resulta señalar que, partimos de que, frente a un caso, puede haber varias soluciones. Sobre el particular, Carrió (1995) señala que en el caso jurídico no hay por lo general una solución, sino varias y que se trata de elegir la más simple, segura, ventajosa y rápida que las circunstancias admitan o consientan.

Siguiendo en línea con lo expuesto, Bain (2004) señala los cinco elementos esenciales que componen ese ambiente natural crítico de aprendizaje, a saber: i. Una pregunta o problema intrigante; ii. La guía en ayudar al estudiantado a entender el significado de la pregunta; iii. Enganchar al alumnado en alguna actividad intelectual de orden mayor: impulsarlos a comparar, aplicar, evaluar, analizar y sintetizar, pero nunca a solo escuchar y recordar. Eso significa a menudo, pedirle al estudiantado hacer y defender juicios y luego proveerles con alguna base para tomar la decisión; iv. Ese ambiente ayuda a cada estudiante a contestar las preguntas. Retarles a desarrollar sus propias explicaciones y comprensiones y a defenderlas; v. Deja a cada estudiante con una pregunta: ¿cuál es la próxima pregunta? Estos cinco elementos, dependiendo del profesorado, pueden aparecer en clases o lecciones interactivas o emergen en sesiones basadas en discusiones o en problemas. En ese sentido, tampoco se trata de desechar por completo las lecciones magistrales, pues estas deben ser vistas como una vía para aclarar y simplificar la materia que es compleja mientras se engranan preguntas retadoras e importantes, o para llamar la atención sobre asuntos importantes, para provocar, para enfocar. Las lecciones magistrales no deben usarse, como una cobertura enciclopédica de algún tema, o como una vía para impresionar al estudiantado. Debe tomarse en cuenta que, en general, el alumnado se siente atraído a pensar mediante problemas, a confrontarlos, a mirar la evidencia, y a razonar más que a memorizar. Lo más importante es que la clase magistral puede ser parte de una exploración mayor, un elemento del ambiente de aprendizaje más que la experiencia total.

\section{ASPECTOS ClAVES PARA EL CAMBIO DE MODELO}

Dentro de los aspectos claves necesarios para ese cambio, podríamos citar los siguientes:

\section{a. El conocimiento se construye, no se recibe}

Como acertadamente señala Bain (2004), la perspectiva tradicional apunta a pensar que la memoria es un compartimento de gran espacio, donde es posible almacenar toda la información deseada.

Tradicionalmente, nos hemos enfocado en la necesidad de que el estudiantado memorice contenidos y los reproduzca durante los exámenes. El problema de este enfoque es que la memoria va sustituyendo contenidos conforme va recibiendo información nueva, con lo que nos enfrentamos con el problema de que 
cada estudiante de Derecho normalmente recordará -en el mejor de los casos- los contenidos de las últimas materias que cursó en la Facultad.

Aunado a lo expuesto y frente al cambio señalado, una pregunta aparentemente paradójica que podría surgir es la siguiente: ¿Cada estudiante debe primero aprender o mejor dicho estudiar los contenidos antes de que pueda pensar sobre ellos?

Al respecto, según Bain (2004) debemos señalar que "...el cerebro humano es una unidad de almacenamiento y de procesamiento a la vez...” (p. 26). Esto significa que pueden procesar y almacenar la información al mismo tiempo, por lo que no necesariamente debe primero estudiar el material respectivo, aunque en la mayoría de las ocasiones sí estimo aconsejable hacer una lectura previa del material respectivo para poder tener una base teorética adecuada al momento de resolver los casos o problemas planteados.

\section{b. Sobre los modelos mentales de la realidad}

Cuando asistimos a la Universidad por primera vez, portamos muchos modelos mentales o esquemas, que usamos o tratamos de usar para comprender las clases o las conferencias que oímos, los textos que leemos, etc.

A criterio de Bain (2004), cuando se encuentra un material nuevo, tratamos de comprenderlo en términos de algo que pensamos que ya conocemos. Eso significa que cuando le hablamos al alumnado, nuestros pensamientos no viajan sin problemas y en forma directa, de nuestros cerebros a los de ellos.

Para lograr lo anterior, resulta fundamental retar intelectualmente al estudiantado. En ese sentido, Bain (2004) señala, por ejemplo, que: “...en lugar de decirles a los estudiantes que están equivocados y luego darles la respuesta correcta, es recomendable hacerle preguntas para ayudarle a ver sus propios errores...” (p. 29).

Resulta también fundamental tomar en cuenta que según Bain (2004) estos modelos mentales cambian lentamente y para lograr ese cambio, las y los estudiantes deben: i. Enfrentar una situación en la cual su modelo mental no funcione (es decir, no le ayuda a explicar o a hacer algo); ii. Preocuparse de que no funciona lo suficientemente bien, para detenerse y lidiar con el tema en cuestión; iii. Ser capaz de manejar el trauma emocional que algunas veces traen los desafíos a las creencias que los han venido acompañando. Por esa razón, estima el autor citado que, debemos retar a nuestros estudiantes intelectualmente. Es decir, crear lo que se denomina la expectativa de fracaso, a saber, ponerlos en aquella situación en la cual, los modelos mentales existentes lleven a expectativas defectuosas, causando que el estudiantado se percate de los problemas que enfrenta al creer en lo que cree.

Para esto, el profesorado debe primero escuchar las concepciones del alumnado antes de retarlo. A este respecto señala Bain (2004) que antes de decirles que están equivocados y de darles las respuestas correctas, es conveniente hacer preguntas para ayudarles a ver sus propios errores.

\section{c. Comprensión de estructuras}

Otro aspecto fundamental para poder llevar a cabo este cambio de modelo, consiste, según Bain (2004) en que cada docente esté en condiciones de que sus estudiantes entiendan estructuras, por ejemplo, cómo las partes individuales se relacionan con el todo y lo más importante, el tipo de decisiones que deben estar en capacidad de tomar con la comprensión que han desarrollado. Ayudarles a construir su entendimiento, es decir, aprender a usar esa información para resolver problemas futuros.

En ese sentido, Perkins (2009) establece siete principios del aprendizaje por todos: 1. Jugar el juego completo; 2 . Hacer que jugar el juego valga la pena; 3 . Trabajar en las partes difíciles; 4. Jugar fuera de la ciudad; 5. Descubrir el juego oculto; 6. Aprender del equipo y de otros equipos; 7. Aprender el juego del aprendizaje. Desde esa perspectiva, Perkins señala en relación con el primer principio citado que, obtener alguna versión del juego completo al inicio tiene sentido, pues da más significado a la iniciativa, así mismo, en relación con el segundo principio indicado, recrear el juego completo aclara porque vale la pena jugarlo, dado que uno ve claramente como las cosas encajan entre sí.

\section{d. Razonamiento por medio de casos o problemas}

Tal y como se ha señalado anteriormente, resulta fundamental poder razonar por medio de la resolución de casos o de problemas. Frente a esto resulta importante tomar en cuenta que, por lo menos, en el plano 
ideal, el Derecho debe ser primordialmente un instrumento para la solución, resolución o replanteamiento de los problemas cotidianos del colectivo social.

Schunk (2012) señala al respecto que la eficacia del aprendizaje basado en problemas tiene sustento en la investigación del cerebro, pues con sus múltiples conexiones, el cerebro humano está diseñado para resolver problemas. Así, el alumnado que colabora en la resolución de problemas descubre nuevas formas de usar y combinar el conocimiento, lo cual crea nuevas conexiones sinápticas.

En este método de resolución de casos o problemas, la formulación de preguntas resulta crucial. Las preguntas nos ayudan a construir conocimiento. En este contexto, contar la historia correcta o formular una pregunta poderosamente provocativa, pueden ayudar enormemente.

Bain (2004) y Capella (2004) señalan que para el aprendizaje innovador, es decir, aquel que consiste en aprender a afrontar problemas y situaciones distintas de las conocidas por quienes enseñan, y a hallarles soluciones inéditas; $y$, secundariamente, consiste en solventar problemas conocidos con soluciones mejores que las dadas, pues son más importantes las preguntas que las respuestas.

El estudio o análisis de casos exige, la mayoría de las veces, según Imbernon y Medina (2006) conocimientos previos de los contenidos relacionados con el caso o el problema a discutir en clase. Lo anterior se puede prever, asignando al estudiante, de previo a que un caso o problema vaya a ser analizado en clase, la lectura y análisis previo de determinados libros o artículos.

Como señala Capella (2004) "Las (buenas clases) son un material con el que construir ese aprendizaje, pero no el único: te han de llevar a los libros, con los que realizarás en solitario una parte sustancial del aprendizaje. Sin ellos estás perdido" (p. 55).

De acuerdo a Bain (2004) "El objetivo consiste en estimular a los estudiantes a que ellos se hagan sus propias preguntas, con eso se estarían poniendo los cimientos para un aprendizaje duradero” (p. 31).

Además del razonamiento mediante problemas o casos, también denominado estudio o método de casos, existen según Imbernon y Medina (2006) otras estrategias metodológicas participativas, tales como: la elaboración de un proyecto; el estudio dirigido; el debate dirigido; la discusión rápida; el foro; la demostración; juegos de rol: dramatización y socio drama; el seminario; el incidente crítico; flash o rueda de intervenciones; diálogos simultáneos; técnica cuatro; cuatro rincones; etc.

Tal y como opinan Imbernon y Medina (2006), la elección de una de esas estrategias depende de varios factores, entre ellos, los objetivos que se persiguen; las dimensiones del grupo, su madurez, el tiempo, la personalidad del y la docente, etc.; y que al aplicar alguna estrategia, debemos conocer sus ventajas e inconvenientes.

\section{e. ¿Qué motiva y qué desmotiva?}

Como señalan Imbernon y Medina (2006) “...uno de los problemas más extendidos en la universidad es la motivación del alumnado..." (p. 117).

¿Qué se puede hacer entonces para motivar al alumnado? Imbernon y Medina (2006) proponen varias soluciones, por ejemplo,

...concretar lo que se espera del alumno, conseguir que el alumno realice cosas por sí mismo; suscitar constantemente la retroalimentación del alumno; invitar al alumno a expresarse; variar la metodología aplicando diferentes estrategias; proponer trabajos en grupos reducidos y valorarlos... (pp. 117-118).

Usar reforzamiento verbal y retroalimentación positiva, en otras palabras, ánimo y elogio puede estimular el interés o, por lo menos, evitar que se evapore. También resulta fundamental alentar la cooperación y la colaboración (Bain, 2004). En ese sentido establece Perkins (2009) como principio básico la importancia de aprender del equipo y de otros equipos, pues cualquier esfuerzo humano es profunda e intrínsecamente colectivo.

En este contexto resulta recomendable evitar usar la obtención de buenas notas para persuadir a las y los estudiantes a estudiar. En vez de eso, Bain (2004) recomienda invocar la materia, las preguntas que surgen, 
y las promesas que hace a cualquier aprendiz. Para este autor, resulta fundamental darle al estudiante algún sentido de control sobre su propia educación. Ayudar a las y los estudiantes a entender la conexión entre los tópicos actuales y una mayor y más profunda investigación, y al hacer eso encontrar bases comunes en esas grandes preguntas que primero motivaron sus propios esfuerzos por aprender. También recomienda el autor citado que, motivar a las y los estudiantes a usar las metodologías, suposiciones y conceptos de una variedad de campos para resolver problemas complejos. En esto resulta fundamental recomendar incorporar literatura de otros campos en su enseñanza y enfatizar en el significado de obtener una educación. En otras palabras, darle importancia al valor de una educación integrada en vez de una fragmentada entre cursos individuales.

Debemos recordar también que, según Capella (2004) “...la enseñanza-aprendizaje es un proceso de carácter gradual y repetitivo. Lo gradual se relaciona con que la transmisión de conocimientos se organiza por pasos..." (p. 31.)

\section{SOBRE EL DESARROLLO DE HABILIDADES Y DE COMPETENCIAS}

Las estrategias citadas en este artículo lo que buscan es precisamente desarrollar en el estudiantado habilidades y competencias que les sean de ayuda en su vida profesional futura. Al respecto señala Pérez (2013) que, la educación basada en el desarrollo de competencias busca desarrollar integralmente habilidades alcanzables mediante estrategias de aprendizaje que produzcan en cada estudiante lo que se ha dado en llamar un aprendizaje autónomo. De acuerdo con Bain (2004), en este modelo “...los estudiantes llegan a ser los actores en el proceso de aprendizaje. Obtienen información, desarrollan su entendimiento y aprenden a usarla.” (p. 52.) El aprendizaje autónomo implica, según Pérez (2013), tomar control personal de este proceso, es decir, cada estudiante autónomo es quien dirige por sí mismo aquellas actividades que están conectadas a su proceso de aprendizaje. Desde esta perspectiva, el rol de la persona docente en las nuevas tendencias pedagógicas permite y fomenta, según Pérez (2013) que, el estudiantado asume su papel como aprendiz autónomo, por lo que, en un enfoque por competencias, los roles del cuerpo docente están más orientados a: ser facilitador, crear situaciones pedagógicas que simulen la vida real de manera que fomenten la cooperación y la interacción, que el alumnado comprenda su papel y asuma su responsabilidad, invitar a la interacción en un discurso claro y conciso, etc.

El profesorado debe hacer hincapié en el diseño de procesos de aprendizaje que permitan al alumnado desarrollar estrategias analíticas y críticas, reflexivas, creativas, aprender a resolver problemas, etc. En una palabra, enseñarle, facilitar el aprendizaje y posibilitar el proceso de aprender a aprender.

$\mathrm{Al}$ respecto Delors (1996) opina que la escuela debe inculcar “...más el gusto y el placer de aprender, la capacidad de aprender a aprender, la curiosidad del intelecto.” (p. 21). Asimismo, este autor señala que, la educación a lo largo de la vida se basa en cuatro pilares: aprender a conocer, aprender a hacer, aprender a vivir juntos y a prender a ser. En relación con los dos primeros, indica Delors (1996):

\footnotetext{
...Aprender a conocer, combinando una cultura genera suficientemente amplia con la posibilidad de profundizar los conocimientos en un pequeño número de materias. Lo que supone, además: aprender a aprender para poder aprovechar las posibilidades que ofrece la educación a lo largo de la vida. Aprender a hacer a fin de adquirir no sólo una calificación profesional sino, más generalmente, una competencia que capacite al individuo para hacer frente a gran número de situaciones y a trabajar en equipo... (p. 109).
}

Así mismo, señala Delors (1996), que aprender a conocer y aprender a hacer son, en gran medida, indisociables, pero lo segundo estás más estrechamente vinculado al tema de la formación profesional.

Así, es el estudiantado quien busca la información, establece nexos significativos con la información ya conocida y sus experiencias previas, y construye conocimientos (los contenidos de aprendizaje) con la ayuda del profesorado. 
A modo de referencia, en Alemania, el $\$ 5^{a}$ párrafo 3, oración 1, de la ley judicial alemana, prevé el término Schlüsselqualifikationen (cualidades o aptitudes clave). Con ese término se ha querido poner énfasis en que la formación del jurista, en general, debe estar más fuertemente orientada a la praxis y al desarrollo de aptitudes claves, tales como: gestión de las negociaciones, manejo del discurso, retórica, técnicas de conciliación, mediación, teoría del interrogatorio y habilidades de comunicación (Paulus, 2003). Para este autor, de estas aptitudes se trata, la mayoría de las veces, de capacidades o habilidades, que uno debe practicar y ejercitar una y otra vez, para que gradualmente se adquieran las técnicas requeridas para sortear los desafíos y nuevas situaciones relacionadas con el trabajo.

\section{SOBRE LA EVALUACIÓN DEL APRENDIZAJE POR HABILIDADES}

El cambio de perspectiva aquí formulado nos plantea un reto importante y es cómo evaluar el aprendizaje en este contexto. Lo coherente sería efectuar un examen mediante el cual el alumnado deba esencialmente resolver casos o problemas, los cuales, para que se califiquen positivamente, no tienen que coincidir con la respuesta que el cuerpo docente estime como correcta, sino que el énfasis estaría puesto en que se haya hecho uso por parte del y la estudiante de una argumentación correcta, valida, lógica y racional. De esta forma, se estaría evaluando el aprendizaje obtenido por el y la estudiantado de esas habilidades y competencias. Así, la evaluación debe consistir, esencialmente, en una verificación y comprobación de las habilidades adquiridas por el estudiantado durante el curso. Esa evaluación debe ir, en todo caso, acorde con los contenidos y los objetivos plasmados en el programa del curso.

La evaluación debe ser vista, asimismo, como un aspecto transversal que se da a lo largo del proceso de enseñanza-aprendizaje y se requiere que el alumnado vea que la evaluación es un instrumento de mejora y no el momento crucial y final de la asignatura en el cual hay que rendir cuentas de lo que ha aprendido o, de lo que no ha aprendido. Que se vea como un elemento positivo, que les permita reflexionar sobre lo que se hace y lo que se puede hacer para mejorarlo (Imbernon y Medina, 2006).

\section{LA PREPARACIÓN DE LAS LECCIONES}

Cada lección requiere por parte de cada docente una preparación y una adaptación a las necesidades y objetivos formulados para el curso. En algunas ocasiones puede ser conveniente una explicación, en otras, formular preguntas, en otras plantear la resolución de casos o problemas, pedirles que escriban algo, que interactúen entre sí, etc.

La preparación o planificación de la clase reviste un aspecto fundamental. En ese sentido, Imbernon y Medina (2006) señalan que la preparación o planificación de la clase hace referencia al hecho de diseñar y concretar en un proyecto, unas intenciones educativas y la forma de conseguirlo, es decir, las actividades que deben ejecutarse. Las actividades representan, de este modo, el punto central del proceso de enseñanzaaprendizaje, y existirían dos grandes tipos de actividades: la del alumnado y la del profesorado.

\section{REFlexiones FinALES}

No se pretende con estas reflexiones dar un conjunto de recomendaciones o recetas para el cambio.

Las ideas aquí expuestas representan un cambio, y es muy normal que el ser humano oponga resistencia a este, pues estamos acostumbrados a actuar en nuestra zona de confort.

$\mathrm{Al}$ inicio de los cursos que he impartido en la Facultad en los últimos años, siempre les he preguntado en cuál curso de Derecho han aprendido más y por qué razón, y un porcentaje muy alto acostumbra a responder que lo ha sido en aquellas clases en las que se utiliza la resolución de casos o de problemas. Esto nos da un 
indicio -siquiera aproximado- de que ese método puede resultar útil en el proceso de enseñanza-aprendizaje de los futuros abogados.

La lección tradicional es aquella en la cual el profesorado expone los contenidos o en el peor de los casos llega a leerlos. Se trata de un contexto unidireccional y lineal. El profesorado recita el contenido y el alumnado debe aprendérselo de memoria, pero ese aprendizaje se olvida y se va sustituyendo por otros contenidos que el estudiantado vaya recibiendo a lo largo de la carrera, de forma tal que lo común es reproducir, al concluir la carrera, el material almacenado en la memoria reciente, es decir, aquellos contenidos que se memorizaron de último.

En esas lecciones tradicionales, se acostumbra a dar uno o más ejemplos, pero a ellos se acude con fines ilustrativos respecto de lo que se está exponiendo.

En este artículo proponemos una modificación en la forma de impartir lecciones en la Facultad de Derecho, de manera tal que se deje de lado, en gran medida, el objetivo de memorizar contenidos y que se busque, más bien, el desarrollo de habilidades y competencias del estudiantado. Para lograr este objetivo, la clase podría iniciar mediante la presentación por parte del profesorado de dos o tres casos o problemas relacionados con la temática o temáticas que se quieran tratar ese día. De esta forma, se le expondrían los casos o problemas al estudiante y se le formularían las preguntas relevantes relacionadas con los casos o problemas, que deben necesariamente representar un reto para el y la estudiante, y que le hagan nacer la curiosidad, que las preguntas los inquieten, les impliquen retos; a partir de ahí, se le puede pedir a las y los estudiantes que traten de resolver los casos o problemas, y conforme van resolviendo los casos, los contenidos se podrán ir desarrollando por parte del profesorado, pero siempre de manera dialéctica e interactiva. Si al dar respuesta a los casos o problemas, el alumnado se equivoca, lo ideal es no hacérselo ver de inmediato, sino hacerle preguntas adicionales para que él mismo se percate de su error.

El proceso educativo de los cursos de la Facultad de Derecho puede plantearse desde la problematización de la enseñanza-aprendizaje, sin que eso signifique que en todos los cursos deba usarse, por ejemplo, el método de resolución de casos.

Haciendo referencia al tema de los programas, señala Haba (1995) que existen materias cuyos programas pueden ser más laxos o variados que los de otras, o donde incluso se puede prescindir de someterlas a un programa fijo; concluyendo el autor citado que no hay ningún tipo de programación aplicable (con sensatez) a todas las materias de la carrera. Lo mismo resultaría aplicable a lo que hemos venido desarrollando en estas líneas. No se pretende, por ejemplo, que en todas las materias de la carrera de Derecho se utilice el método de resolución de casos o problemas, aunque sí puede ser conveniente, por ejemplo, que en todas se dé un ambiente natural crítico de aprendizaje, con todo lo que ello conlleva. Es decir, cada profesor y profesora debería definir, dependiendo de la materia que imparta, qué aspectos de los que aquí se desarrollan, le pueden ser de utilidad pedagógica en el respectivo curso.

$\mathrm{Al}$ respecto señala Haba (1995):

...Lo que vale no es sino aquello que el profesor consiga hace 'germinar' efectivamente en el espíritu de los alumnos. Pero esto depende, antes que nada, de cuanto pueda 'germinar' en la cabeza del profesor mismo, lo cual sobre todo surge como resultado de sus investigaciones personales. La verdadera enseñanza no consiste en provocar la memorización de 'bloques', sino en comunicar a los alumnos un saber que está en movimiento; y sobre esto, nadie puede dar mejor testimonio que alguien que participa en ese 'movimiento', o sea, cuanto el profesor pueda transmitir sobre sus propias investigaciones... (p. 154).

Este cambio en la perspectiva implica también potenciar, entre otras habilidades, la oralidad, como destreza fundamental que deben dominar los y las alumnas en estos tiempos. Se echa mano de la oralidad cuando mediante la dinámica expuesta, no sólo el profesorado es el que debe hacer uso de la palabra, sino que se incentiva a que todo el alumnado aprenda paulatinamente a expresarse oralmente.

Igualmente, en el modelo expuesto, se pueden incluir las TIC, como herramienta fundamental para lograr generar ese ambiente natural crítico de aprendizaje, al que se aludió anteriormente. 
Resulta necesario dejar atrás el enfoque unidireccional en que se han impartido las lecciones en la Facultad de Derecho, para optar por un proceso de enseñanza-aprendizaje dinámico, recíproco, bidireccional, en el que el alumnado asuma un rol activo y participativo, es decir, se constituya en un estudiante autónomo, con plena conciencia de sus responsabilidades y obligaciones; del mismo modo, que el profesorado tenga claro cuáles son sus obligaciones para fomentar el citado proceso.

\section{REFERENCIAS}

Arias, M. y Francis, S. y Marín, P. (2012). Modelos Pedagógicos de formación docente en la Universidad de Costa Rica. Precisiones, historia y desafíos. Costa Rica: Departamento de Docencia Universitaria, Escuela de Formación Docente Universidad de Costa Rica.

Bain, K. (2004). What the Best College Teacher do. Cambridge: Harvard University Press.

Capella, J.R. (2004). El aprendizaje del aprendizaje. Una introducción al estudio del Derecho. Madrid: Editorial Trotta.

Carrió, G. (1995). Cómo estudiar y cómo argumentar un caso. Consejos elementales para abogados jóvenes. Buenos Aires: Editorial Abeledo-Perrot.

De Alcántara, P. (1999). Compendio de pedagogía teórico-práctica. Alicante: Biblioteca Virtual Miguel de Cervantes.

Delors, J. (1996). La educación encierra un tesoro. España: Grupo Santillana de Ediciones.

Haba, P. (1995). Pedagogismo y "Mala fe". Costa Rica: Investigaciones Jurídicas, S.A.

Imbernon, F. y Medina, J. L. (2006). Metodología participativa en el aula universitaria. La participación del alumnado. En M. Martínez y S. Carrasco (Coords.), Propuestas para el cambio docente en la universidad. (pp. 91-121). Barcelona: Ediciones Octaedro.

Paulus, C. (2003), Schlüsselqualifikationen im Studieninhalt. En: V. Römermann y C. Paulus. (Eds.). Schlüsselqualifikationenfür Jurastudium, Examen und Beruf. Ein Lebrbuch (pp. 1-32). Múnich, Alemania: Editorial C.H.Beck.

Pausch, R. (2008). The last Lecture. Nueva York: Hyperion.

Pérez, L. (2013). El rol del docente en el aprendizaje autónomo: la perspectiva del estudiante y la relación con su rendimiento académico. Revista Repositorio Digital de la Ciencia y Cultura de El Salvador, 7 (11), 45-62.

Perkins, D. (2009). Making Learning Whole: How seven principles of teaching can transform education. San Francisco, California: Jossey-Bass.

Picardo, O., Escobar, J. y Balmore, R. (2005). Diccionario Enciclopédico de Ciencias de la Educación. San Salvador, El Salvador: Centro de Investigación Educativa, Colegio García Flamenco.

Real Academia. (1994). Diccionario de la Lengua Española (Vigésima Primera Edición). Madrid: Real Academia Española, RAE

Ruiz, L. (1904). Tratado elemental de Pedagogía. México: Herrero Hermanos, Editores.

Schunk D., (2012). Teorías del aprendizaje: una perspectiva educativa, México: Pearson Educación.

\section{BY-NC-ND}

